\title{
Novel copolymers of vinyl acetate. 2. Oxy ring-substituted ethyl 2-cyano-
}

\section{3-phenyl-2-propenoates}

Lisa Barilla, Terry L. Bogatay, Joy A. Davis, James R. Fienberg, Christopher E. Jensen, Karen S. Johnson, Poonam K. Kahlon, Meeah Loveless, Karim N. Sajwani, Maria A. Tsarpralis, Amy T. Stevens, Peter Tessalee, Christopher M. Varona, Thanarat Viriyakul, Elizabeth A. Wadey, Michael L. Wills, Emily E. Wroblewski and Gregory B. Kharas

DePaul University, Chemistry and Biochemistry Department, 1110 West Belden Avenue, Chicago, IL 60614-3214

Novel copolymers of vinyl acetate and oxy ring-substituted ethyl 2-cyano-3-phenyl-2-

propenoates, $\mathrm{RPhCH}=\mathrm{C}(\mathrm{CN}) \mathrm{CO}_{2} \mathrm{C}_{2} \mathrm{H}_{5}$ (where $\mathrm{R}$ is 2-methoxy, 3-methoxy, 4-methoxy, 2ethoxy, 4-ethoxy, 4-propoxy, 4-butoxy, 2,3-dimethoxy, 2,4-dimethoxy, 2,5-dimethoxy, 3,4dimethoxy, 2,3,4-trimethoxy, 2,4,5-trimethoxy, 2,4,6-trimethoxy, 3,4,5-trimethoxy) were prepared in solution with radical initiation at $70^{\circ} \mathrm{C}$. The propenoates were synthesized by the piperidine catalyzed Knoevenagel condensation of ring-substituted benzaldehydes and ethyl cyanoacetate, and characterized by $\mathrm{CHN}$ analysis, IR, ${ }^{1} \mathrm{H}$ and ${ }^{13} \mathrm{C}-\mathrm{NMR}$. The compositions of the copolymers were calculated from nitrogen analysis and the structures were analyzed by IR, ${ }^{1} \mathrm{H}$ and ${ }^{13} \mathrm{C}-\mathrm{NMR}$. Thermal behavior of the copolymers was studied by DSC (Tg) and 
TGA. Decomposition of the copolymers in nitrogen occurred in two steps, first in the 160$350^{\circ} \mathrm{C}$ range with residue $(2.2-25.3 \mathrm{wt} \%)$, which then decomposed in the $500-650^{\circ} \mathrm{C}$ range.

\section{Introduction}

Ethyl oxy ring-substituted esters of 2-cyano-3-phenyl-2-propenoic acid (EOCP), $\mathrm{R}^{1} \mathrm{PhCH}=\mathrm{C}(\mathrm{CN}) \mathrm{CO}_{2} \mathrm{C}_{2} \mathrm{H}_{5}$ continue to attract attention as compounds with useful properties and as comonomers for functionalization of commercial polymers. There is report on cistrans isomerization of 2-methoxy ring substituted EOCP by various nucleophiles [1]. Effect of 3-methoxy ring-substituted EOCP was studied in glutathione-S-transferase activity in Setaria cervi females [2]. 4-Methoxy ring-substituted EOCP was utilized in synthesis of benzo[c]coumarin and of benzo[c]pyrano[3,2-c]quinoline derivatives [3]. 2-Ethoxy ringsubstituted EOCP was used in organic crystals for fabrication of electroluminescent devices [4]. 4-Ethoxy ring-substituted EOCP was cited in studies of stereochemistry of interaction of pyridinium ylides with $\alpha, \beta$-unsaturated nitriles [5]. 4-Propoxy ring-substituted EOCP was involved in sustainable C-C bond formation through Knoevenagel reaction catalyzed by MgO-based catalysts [6]. Ethyl p-butoxycinnamate was applied in maintaining self-renewal and pluripotency of stem cells [7]. 4-Phenoxy-substituted EOCP was used in DBU-mediated $[4+2]$ annulations of donor-acceptor cyclopropanes with 3-aryl-2-cyanoacrylates for the synthesis of fully substituted anilines [8], as well as in preparation of azaindazoles as Btk kinase modulators [9]. Copolymers of oxy aryl-substituted EOCP with styrene were prepared earlier $[10,11]$. 2,3-Dimethoxy ring-substituted EOCP was involved in highly efficient synthesis of pyranoquinoline derivatives catalyzed by piperidine [12]. 2,4- 
Dimethoxy EOCP was involved design, synthesis and anticancer activity of novel benzothiazole analogues [13]. 2,5-Dimethoxy EOCP was used in synthesis and antimicrobial activity of some novel hydrazide, benzochromenone, dihydropyridine, pyrrole, thiazole and thiophene derivatives [14]. 3,4-Dimethoxy EOCP was involved in synthesis, biological evaluation and in Silico ADME-T study of novel polyfunctional pyridines as anticancer and antioxidant agents [15]. 2,3,4-Trimethoxy EOCP were part of synthesis and biological screening of novel 3-amino-4-arylidene-5-pyrazolones and thiazolo[3,2-a] pyrimidines [16]. 2,4,5-Trimethoxy EOCP used in studies of antitumor activity of novel pyridine, thiophene and thiazole derivatives [17]. Synthesis, characterization and heterogeneous base catalysis of amino functionalized lanthanide metal-organic frameworks are related to 2,4,6-trimethoxy EOCP [18], whereas the report on synthesis, antimicrobial and antitumor study of new pyrido[2,1-a]isoquinolines via isoquinoline-1-acetonitrile involves 3,4,5-trimethoxy EOCP [19].

In continuation of our study of copolymers of vinyl acetate and oxy aryl-substituted esters 2-cyano-3-phenyl-2-propenoic acid, we have prepared oxy ring-substituted ethyl 2cyano-3-phenyl-2-propenoates, $\mathrm{RPhCH}=\mathrm{C}(\mathrm{CN}) \mathrm{CO}_{2} \mathrm{C}_{2} \mathrm{H}_{5}$, where $\mathrm{R}$ is 2-methoxy, 3methoxy, 4-methoxy, 2-ethoxy, 4-ethoxy, 4-propoxy, 4-butoxy, 2,3-dimethoxy, 2,4dimethoxy, 2,5-dimethoxy, 3,4-dimethoxy, 2,3,4-trimethoxy, 2,4,5-trimethoxy, 2,4,6trimethoxy, 3,4,5-trimethoxy, and copolymerized with vinyl acetate. 
There are reports of syntheses of 2-methoxy, 3-methoxy, 4-methoxy [20, 21], 2ethoxy [22], 4-ethoxy, 4-propoxy, 4-butoxy [23], 2,3-dimethoxy, 2,4-dimethoxy, 2,5dimethoxy, 3,4-dimethoxy, 2,3,4-trimethoxy, 2,4,5-trimethoxy, 2,4,6-trimethoxy, 3,4,5trimethoxy [24] ring-substituted EOCP. To the best of our knowledge, there have been no reports on copolymerization of these ethyl 2-cyano-3-phenyl-2-propenoates with vinyl acetate [25].

\section{Experimental}

2-Methoxy, 3-methoxy, 4-methoxy, 2-ethoxy, 4-ethoxy, 4-propoxy, 4-butoxy, 2,3dimethoxy, 2,4-dimethoxy, 2,5-dimethoxy, 3,4-dimethoxy, 2,3,4-trimethoxy, 2,4,5trimethoxy, 2,4,6-trimethoxy, 3,4,5-trimethoxy-substituted benzaldehydes, ethyl cyanoacetate, piperidine, vinyl acetate, 1,1'-azobiscyclohexanecarbonitrile, $(\mathrm{ABCN})$, and toluene supplied from Sigma-Aldrich Co., were used as received. Instrumentation is described in [26].

\section{Synthesis of Monomers}

The ring-substituted ethyl 2-cyano-3-phenyl-2-propenoates (EOCP) were synthesized by Knoevenagel condensation [27] of a ring-substituted benzaldehyde with ethyl cyanoacetate, catalyzed by base, piperidine. The preparation procedure was essentially the same for all the monomers [26].

$$
\mathrm{RPhCHO}+\mathrm{NCCH}_{2} \mathrm{CO}_{2} \mathrm{C}_{2} \mathrm{H}_{5} \rightarrow \mathrm{RPhCH}=\mathrm{C}(\mathrm{CN}) \mathrm{CO}_{2} \mathrm{C}_{2} \mathrm{H}_{5}
$$

where R is 2-methoxy, 3-methoxy, 4-methoxy, 2-ethoxy, 4-ethoxy, 4-propoxy, 4-butoxy, 2,3-dimethoxy, 2,4-dimethoxy, 2,5-dimethoxy, 3,4-dimethoxy, 2,3,4-trimethoxy, 2,4,5trimethoxy, 2,4,6-trimethoxy, 3,4,5-trimethoxy. 


\subsection{Ethyl 2-cyano-3-(2-methoxyphenyl)-2-propenoate}

Yield 76\%; mp $79.7^{\circ} \mathrm{C},{ }^{1} \mathrm{H}-\mathrm{NMR} \delta 8.6(\mathrm{~s}, 1 \mathrm{H}, \mathrm{CH}=), 8.2-7.5(\mathrm{~m}, 4 \mathrm{H}, \mathrm{Ph}), 4.3(\mathrm{q}, 2 \mathrm{H}$, $\left.\mathrm{OCH}_{2}\right), 3.8\left(\mathrm{~s}, 3 \mathrm{H}, \mathrm{PhOCH}_{3}\right), 1.3\left(\mathrm{t}, 3 \mathrm{H}, \mathrm{OCH}_{2} \underline{\mathrm{H}}_{3}\right) ;{ }^{13} \mathrm{C}-\mathrm{NMR} \delta 163(\mathrm{C}=\mathrm{O}), 152(\mathrm{HC}=)$, 148, 131, 130, 122, $111(\mathrm{Ph}), 116(\mathrm{CN}), 111(\mathrm{C}=), 61\left(\mathrm{OCH}_{2}\right), 56\left(\mathrm{PhOCH}_{3}\right), 14$ $\left(\mathrm{OCH}_{2} \underline{\mathrm{CH}}_{3}\right)$; IR $\left(\mathrm{cm}^{-1}\right)$ : 3023-2832 (m, C-H), 2224 (m, CN), 1742 (s, C=O), $1574(\mathrm{C}=\mathrm{C})$, 1234 (s, C-O-CH 3 ), 812 (s, C-H out of plane). Anal. Calcd. for $\mathrm{C}_{13} \mathrm{H}_{13} \mathrm{NO}_{3}: \mathrm{C}, 67.52 ; \mathrm{H}$, 5.67; N, 6.06; Found: C, 69.79; H, 6.09; N, 5.53.

\subsection{Ethyl 2-cyano-3-(3-methoxylphenyl)-2-propenoate}

Yield $84 \%$; mp $207.6^{\circ} \mathrm{C},{ }^{1} \mathrm{H}-\mathrm{NMR} \delta 8.2(\mathrm{~s}, 1 \mathrm{H}, \mathrm{CH}=), 7.6-6.7(\mathrm{~m}, 4 \mathrm{H}, \mathrm{Ph}), 4.3(\mathrm{q}, 2 \mathrm{H}$, $\left.\mathrm{OCH}_{2}\right), 3.8\left(\mathrm{~s}, 3 \mathrm{H}, \mathrm{PhOCH}_{3}\right), 1.3\left(\mathrm{t}, 3 \mathrm{H}, \mathrm{OCH}_{2} \underline{\mathrm{H}}_{3}\right) ;{ }^{13} \mathrm{C}-\mathrm{NMR} \delta 163(\mathrm{C}=\mathrm{O}), 154(\mathrm{HC}=)$, 160, 133, 130, $114(\mathrm{Ph}), 116(\mathrm{CN}), 103(\mathrm{C}=), 61\left(\mathrm{OCH}_{2}\right), 55\left(\mathrm{PhOCH}_{3}\right), 14\left(\mathrm{OCH}_{2} \underline{C H}_{3}\right)$; IR (cm $\left.{ }^{-1}\right):$ 3038-2831 (m, C-H), $2222(\mathrm{~m}, \mathrm{CN}), 1721(\mathrm{~s}, \mathrm{C}=\mathrm{O}), 1580(\mathrm{C}=\mathrm{C}), 1222(\mathrm{~s}, \mathrm{C}-$ O-CH 3 ), 843 (s, C-H out of plane). Anal. Calcd. for $\mathrm{C}_{13} \mathrm{H}_{13} \mathrm{NO}_{3}: \mathrm{C}, 67.52 ; \mathrm{H}, 5.67$; , 6.06; Found: C, 65.29; H, 5.83; N, 6.01.

\subsection{Ethyl 2-cyano-3-(4-methoxyphenyl)-2-propenoate}

Yield $89 \%$; mp $85.3^{\circ} \mathrm{C},{ }^{1} \mathrm{H}-\mathrm{NMR} \delta 8.3(\mathrm{~s}, 1 \mathrm{H}, \mathrm{CH}=), 7.5,6.9$ (d, 4H, Ph), 4.3 (q, 2H, $\left.\mathrm{OCH}_{2}\right), 3.8\left(\mathrm{t}, 3 \mathrm{H}, \mathrm{PhOCH}_{3}\right), 1.3\left(\mathrm{t}, 3 \mathrm{H}, \mathrm{OCH}_{2} \mathrm{C}_{3}\right) ;{ }^{13} \mathrm{C}-\mathrm{NMR} \delta 163(\mathrm{C}=\mathrm{O}), 154(\mathrm{HC}=)$, 145, 125, 131, $114(\mathrm{Ph}), 116(\mathrm{CN}), 100(\mathrm{C}=), 61\left(\mathrm{OCH}_{2}\right), 55\left(\mathrm{PhOCH}_{3}\right), 14\left(\mathrm{OCH}_{2} \underline{\mathrm{CH}}_{3}\right)$; IR (cm $\left.{ }^{-1}\right)$ : 3029-2921 (m, C-H), $2223(\mathrm{~m}, \mathrm{CN}), 1741$ (s, C=O), 1239 (s, C-O-C), 848 (s, C-H out of plane). Anal. Calcd. for $\mathrm{C}_{13} \mathrm{H}_{13} \mathrm{NO}_{3}$ : C, 67.52; H, 5.67; N, 6.06; Found: C, 67.43; H, 5.70; N, 6.04. 


\subsection{Ethyl 2-cyano-3-(2-ethoxyphenyl)-2-propenoate}

Yield 74\%; mp 86. ${ }^{\circ} \mathrm{C},{ }^{1} \mathrm{H}-\mathrm{NMR} \delta 8.2(\mathrm{~s}, 1 \mathrm{H}, \mathrm{CH}=), 7.5-6.8(\mathrm{~m}, 4 \mathrm{H}, \mathrm{Ph}), 4.3(\mathrm{q}, 2 \mathrm{H}$, $\left.\mathrm{OCH}_{2}\right), 4.1$ (q, 2H, $\left.\mathrm{PhOCH}_{2}\right), 1.3\left(\mathrm{t}, 3 \mathrm{H}, \mathrm{OCH}_{2} \mathrm{CH}_{3}\right), 1.3$ (t, 3H, $\left.\mathrm{PhOCH}_{2} \mathrm{CH}_{3}\right) ;{ }^{13} \mathrm{C}-\mathrm{NMR}$ $\delta 163(\mathrm{C}=\mathrm{O}), 152(\mathrm{HC}=), 153,131,130,122,112(\mathrm{Ph}), 116(\mathrm{CN}), 111(\mathrm{C}=), 64$ $\left(\mathrm{PhOCH}_{2}\right), 61\left(\mathrm{OCH}_{2}\right), 15\left(\mathrm{PhCH}_{2} \underline{\mathrm{CH}_{3}}\right), 14\left(\mathrm{OCH}_{2} \underline{\mathrm{CH}_{3}}\right) ; \mathrm{IR}\left(\mathrm{cm}^{-1}\right): 3123-2824(\mathrm{~m}, \mathrm{C}-$ H), 2222 (m, CN), 1723 (s, C=O), 1617 (C=C), 1242 (s, C-O-C), 849 (s, C-H out of plane). Anal. Calcd. for $\mathrm{C}_{14} \mathrm{H}_{15} \mathrm{NO}_{3}$ : C, 68.56; H, 6.16; N, 5.71; Found: C, 68.89; H, 6.19; N, 5.76.

\subsection{Ethyl 2-cyano-3-(4-ethoxyphenyl)-2-propenoate}

Yield $87 \%$; mp $94.5^{\circ} \mathrm{C},{ }^{1} \mathrm{H}-\mathrm{NMR} \delta 8.3(\mathrm{~s}, 1 \mathrm{H}, \mathrm{CH}=), 7.6-6.8(\mathrm{~m}, 4 \mathrm{H}, \mathrm{Ph}), 4.3(\mathrm{q}, 2 \mathrm{H}$, $\left.\mathrm{OCH}_{2}\right), 4.1$ (q, 2H, $\left.\mathrm{PhOCH}_{2}\right), 1.3\left(\mathrm{t}, 3 \mathrm{H}, \mathrm{OCH}_{2} \mathrm{CH}_{3}\right), 1.3\left(\mathrm{t}, 3 \mathrm{H}, \mathrm{PhOCH}_{2} \mathrm{CH}_{3}\right) ;{ }^{13} \mathrm{C}-\mathrm{NMR}$ $\delta 163(\mathrm{C}=\mathrm{O}), 154(\mathrm{HC}=), 151,131,125,115(\mathrm{Ph}), 116(\mathrm{CN}), 100(\mathrm{C}=), 64\left(\mathrm{PhOCH}_{2}\right), 61$ $\left(\mathrm{OCH}_{2}\right), 15\left(\mathrm{PhCH}_{2} \underline{\mathrm{CH}}_{3}\right), 14\left(\mathrm{OCH}_{2} \underline{\mathrm{CH}_{3}}\right) ; \mathrm{IR}\left(\mathrm{cm}^{-1}\right): 3029-2854(\mathrm{~m}, \mathrm{C}-\mathrm{H}), 2224$ (m, CN), 1735 (s, C=O), 1612 (C=C), 1248 (s, C-O-C), 845 (s, C-H out of plane). Anal. Calcd. for $\mathrm{C}_{14} \mathrm{H}_{15} \mathrm{NO}_{3}$ : C, 68.56; H, 6.16; N, 5.71; Found: C, 68.62; H, 6.11; N, 5.57.

\subsection{Ethyl 2-cyano-3-(4-propoxyphenyl)-2-propenoate}

Yield 79\%; mp $86.9^{\circ} \mathrm{C} ;{ }^{1} \mathrm{H}-\mathrm{NMR} \delta 8.2$ (s, $\left.1 \mathrm{H}, \mathrm{CH}=\right), 7.5,6.9(\mathrm{~m}, 4 \mathrm{H}, \mathrm{Ph}), 4.3$ (q, 2H, $\left.\mathrm{OCH}_{2}\right), 3.9$ (t, 2H, $\left.\mathrm{PhOCH}_{2}\right), 1.7\left(\mathrm{~m}, 2 \mathrm{H}, \mathrm{PhOCH}_{2} \underline{\mathrm{C}}_{2}\right), 1.3\left(\mathrm{~m}, 12 \mathrm{H}, \mathrm{CH}_{3}\right), 1.0(\mathrm{t}, 3 \mathrm{H}$, $\left.\mathrm{PhOCH}_{2} \mathrm{CH}_{2} \mathrm{CH}_{3}\right) ;{ }^{13} \mathrm{C}-\mathrm{NMR} \delta 163(\mathrm{C}=\mathrm{O}), 154(\mathrm{HC}=), 152,125,131,115(\mathrm{Ph}), 116$ (CN), $100(\mathrm{C}=), 70\left(\mathrm{PhCH}_{2}\right), 61\left(\mathrm{OCH}_{2}\right), 23\left(\mathrm{PhOCH}_{2} \underline{\mathrm{CH}}_{2}\right), 14\left(\mathrm{OCH}_{2} \underline{\mathrm{CH}}_{3}\right), 10$ $\left(\mathrm{PhOCH}_{2} \mathrm{CH}_{2} \mathrm{CH}_{3}\right)$; IR $\left(\mathrm{cm}^{-1}\right)$ : 3005-2887 (m, C-H), 2224 (m, CN), 1724 (s, C=O), 1582 
(C=C), 1220 (s, C-O-C), 811, 753 (s, C-H out of plane). Anal. Calcd. for $\mathrm{C}_{15} \mathrm{H}_{17} \mathrm{NO}_{3}$ : C,

69.48; H, 6.61; N, 5.40; Found: C, 69.47; H, 6.57; N, 5.30.

\subsection{Ethyl 2-cyano-3-(4-butoxyphenyl)-2-propenoate}

Yield $81 \%$; mp $73.3^{\circ} \mathrm{C} ;{ }^{1} \mathrm{H}-\mathrm{NMR} \delta 8.2(\mathrm{~s}, 1 \mathrm{H}, \mathrm{CH}=), 7.5,6.9(\mathrm{~m}, 4 \mathrm{H}, \mathrm{Ph}), 4.3(\mathrm{q}, 2 \mathrm{H}$, $\left.\mathrm{OCH}_{2}\right), 4.0\left(\mathrm{t}, 2 \mathrm{H}, \mathrm{PhOCH}_{2}\right), 1.7\left(\mathrm{~m}, 2 \mathrm{H}, \mathrm{PhOCH}_{2} \underline{\mathrm{C}}_{2}\right), 1.4\left(\mathrm{~m}, \mathrm{PhOCH}_{2} \mathrm{CH}_{2} \mathrm{C}_{2}\right), 1.3$ $\left(\mathrm{t}, 3 \mathrm{H}, \mathrm{OCH}_{2} \underline{\mathrm{C}}_{3}\right), 0.9\left(\mathrm{t}, 3 \mathrm{H}, \mathrm{PhO}\left(\mathrm{CH}_{2}\right)_{3} \mathrm{C}_{3}\right) ;{ }^{13} \mathrm{C}-\mathrm{NMR} \delta 163(\mathrm{C}=\mathrm{O}), 154(\mathrm{HC}=), 152$, 131, 125, 126, $115(\mathrm{Ph}), 116(\mathrm{CN}), 100(\mathrm{C}=), 68\left(\mathrm{PhOCH}_{2}\right), 61\left(\mathrm{OCH}_{2}\right), 31$

$\left(\mathrm{PhOCH}_{2} \underline{\mathrm{CH}}_{2}\right), 19\left(\mathrm{PhOCH}_{2} \mathrm{CH}_{2} \underline{\mathrm{CH}}_{2}\right), 14\left(\mathrm{OCH}_{2} \underline{\mathrm{CH}_{3}}\right), 13\left(\mathrm{PhO}\left(\mathrm{CH}_{2}\right)_{3} \underline{\mathrm{CH}}_{3}\right) ; \mathrm{IR}\left(\mathrm{cm}^{-1}\right)$ : 3026-2822 (m, C-H), 2223 (m, CN), 1744 (s, C=O), 1576 (C=C), 1221 (s, C-O-C), 845 (s, C-H out of plane). Anal. Calcd. for $\mathrm{C}_{16} \mathrm{H}_{19} \mathrm{NO}_{3}$ : C, 70.31; H, 7.01; N, 5.12; Found: C, 58.02; H, 3.68; N, 5.11.

\subsection{Ethyl 2-cyano-3-(2,3-dimethoxylphenyl)-2-propenoate}

Yield 76\%; mp $120^{\circ} \mathrm{C},{ }^{1} \mathrm{H}-\mathrm{NMR} \delta 8.1(\mathrm{~s}, 1 \mathrm{H}, \mathrm{CH}=), 7.5-6.8(\mathrm{~m}, 3 \mathrm{H}, \mathrm{Ph}), 4.3(\mathrm{q}, 2 \mathrm{H}$, $\left.\mathrm{OCH}_{2}\right), 3.8\left(\mathrm{~s}, 6 \mathrm{H}, \mathrm{PhOCH}_{3}\right), 1.3\left(\mathrm{t}, 3 \mathrm{H}, \mathrm{OCH}_{2} \underline{\mathrm{C}}_{3}\right) ;{ }^{13} \mathrm{C}-\mathrm{NMR} \delta 163(\mathrm{C}=\mathrm{O}), 152(\mathrm{HC}=)$, 151, 127, 126, $121(\mathrm{Ph}), 116(\mathrm{CN}), 111(\mathrm{C}=), 61\left(\mathrm{OCH}_{2}\right), 56\left(\mathrm{PhOCH}_{3}\right), 14\left(\mathrm{OCH}_{2} \underline{\mathrm{CH}}_{3}\right)$; IR ( $\left.\mathrm{cm}^{-1}\right)$ : 3021-2838 (m, C-H), $2223(\mathrm{~m}, \mathrm{CN}), 1724(\mathrm{~s}, \mathrm{C}=\mathrm{O}), 1585(\mathrm{C}=\mathrm{C}), 1232(\mathrm{~s}, \mathrm{C}-$ O-CH 3 ), 847 (s, C-H out of plane). Anal. Calcd. for $\mathrm{C}_{14} \mathrm{H}_{15} \mathrm{NO}_{4}$ : C, 64.36; H, 5.79; N, 5.36; Found: C, 64.41; H, 5.66; N, 5.29.

\subsection{Ethyl 2-cyano-3-(2,4-dimethoxylphenyl)-2-propenoate}

Yield 89\%; mp $146^{\circ} \mathrm{C},{ }^{1} \mathrm{H}-\mathrm{NMR} \delta 8.1$ (s, $\left.1 \mathrm{H}, \mathrm{CH}=\right), 7.6-6.8(\mathrm{~m}, 3 \mathrm{H}, \mathrm{Ph}), 4.3$ (q, $2 \mathrm{H}$, $\left.\mathrm{OCH}_{2}\right), 3.8\left(\mathrm{~s}, 6 \mathrm{H}, \mathrm{PhOCH}_{3}\right), 1.3\left(\mathrm{t}, 3 \mathrm{H}, \mathrm{OCH}_{2} \underline{\mathrm{CH}}_{3}\right) ;{ }^{13} \mathrm{C}-\mathrm{NMR} \delta 163(\mathrm{C}=\mathrm{O}), 152(\mathrm{HC}=)$, 
151, 131, 107, 106, $98(\mathrm{Ph}), 116(\mathrm{CN}), 97(\mathrm{C}=), 61\left(\mathrm{OCH}_{2}\right)$, 56, $55\left(\mathrm{PhOCH}_{3}\right), 14$

$\left(\mathrm{OCH}_{2} \underline{\mathrm{CH}}_{3}\right)$; IR $\left(\mathrm{cm}^{-1}\right)$ : 3022-2844 (m, C-H), 2223 (m, CN), 1729 (s, C=O), 1567 (C=C), 1237 (s, C-O-CH3), 842 (s, C-H out of plane). Anal. Calcd. for $\mathrm{C}_{14} \mathrm{H}_{15} \mathrm{NO}_{4}$ : C, 64.36; H, 5.79; N, 5.36; Found: C, 64.70; H, 5.69; N, 5.28.

\subsection{Ethyl 2-cyano-3-(2,5-dimethoxylphenyl)-2-propenoate}

Yield $86 \%$; mp $86.8^{\circ} \mathrm{C},{ }^{1} \mathrm{H}-\mathrm{NMR} \delta 8.2$ (s, $\left.1 \mathrm{H}, \mathrm{CH}=\right), 7.1-6.5(\mathrm{~m}, 3 \mathrm{H}, \mathrm{Ph}), 4.3(\mathrm{q}, 2 \mathrm{H}$, $\left.\mathrm{OCH}_{2}\right), 3.8\left(\mathrm{~s}, 6 \mathrm{H}, \mathrm{PhOCH}_{3}\right), 1.3\left(\mathrm{t}, 3 \mathrm{H}, \mathrm{OCH}_{2} \mathrm{CH}_{3}\right) ;{ }^{13} \mathrm{C}-\mathrm{NMR} \delta 163(\mathrm{C}=\mathrm{O}), 152(\mathrm{HC}=)$, 151, 121, 114, $113(\mathrm{Ph}), 116(\mathrm{CN}), 111(\mathrm{C}=), 61\left(\mathrm{OCH}_{2}\right), 56\left(\mathrm{PhOCH}_{3}\right), 14\left(\mathrm{OCH}_{2} \underline{\mathrm{CH}}_{3}\right)$; IR (cm $\left.{ }^{-1}\right): 3001-2839(\mathrm{~m}, \mathrm{C}-\mathrm{H}), 2225(\mathrm{~m}, \mathrm{CN}), 1728(\mathrm{~s}, \mathrm{C}=\mathrm{O}), 1582(\mathrm{C}=\mathrm{C}), 1233(\mathrm{~s}, \mathrm{C}-$ O-CH 3 ), 849 (s, C-H out of plane). Anal. Calcd. for $\mathrm{C}_{14} \mathrm{H}_{15} \mathrm{NO}_{4}$ : C, 64.36; H, 5.79; N, 5.36; Found: C, 64.37; H, 5.81; N, 5.38.

\subsection{Ethyl 2-cyano-3-(3,4-dimethoxylphenyl)-2-propenoate}

Yield 79\%; mp $157.1^{\circ} \mathrm{C},{ }^{1} \mathrm{H}-\mathrm{NMR} \delta 8.2$ (s, $\left.1 \mathrm{H}, \mathrm{CH}=\right), 7.6-6.8(\mathrm{~m}, 3 \mathrm{H}, \mathrm{Ph}), 4.3(\mathrm{q}, 2 \mathrm{H}$, $\left.\mathrm{OCH}_{2}\right), 3.8\left(\mathrm{~s}, 6 \mathrm{H}, \mathrm{PhOCH}_{3}\right), 1.3\left(\mathrm{t}, 3 \mathrm{H}, \mathrm{OCH}_{2} \underline{\mathrm{C}}_{3}\right) ;{ }^{13} \mathrm{C}-\mathrm{NMR} \delta 163(\mathrm{C}=\mathrm{O}), 154(\mathrm{HC}=)$, 153, 149, 127, 126, $123(\mathrm{Ph}), 116(\mathrm{CN}), 100(\mathrm{C}=), 61\left(\mathrm{OCH}_{2}\right), 56\left(\mathrm{PhOCH}_{3}\right), 14$ $\left(\mathrm{OCH}_{2} \underline{\mathrm{CH}}_{3}\right)$; IR $\left(\mathrm{cm}^{-1}\right): 3011-2828$ (m, C-H), 2224 (m, CN), $1722(\mathrm{~s}, \mathrm{C}=\mathrm{O}), 1584(\mathrm{C}=\mathrm{C})$, 1233 (s, C-O-CH 3 ), 843 (s, C-H out of plane). Anal. Calcd. for $\mathrm{C}_{14} \mathrm{H}_{15} \mathrm{NO}_{4}$ : C, 64.36; H, 5.79; N, 5.36; Found: C, 64.33; H, 5.71; N, 5.28.

\subsection{Ethyl 2-cyano-3-(2,3,4-trimethoxylphenyl)-2-propenoate}

Yield 69\%; mp $113.9^{\circ} \mathrm{C},{ }^{1} \mathrm{H}-\mathrm{NMR} \delta 8.2(\mathrm{~s}, 1 \mathrm{H}, \mathrm{CH}=), 7.5,6.9(\mathrm{~m}, 2 \mathrm{H}, \mathrm{Ph}), 4.3(\mathrm{q}, 2 \mathrm{H}$, $\left.\mathrm{OCH}_{2}\right), 3.8\left(\mathrm{~s}, 9 \mathrm{H}, \mathrm{PhOCH}_{3}\right), 1.3\left(\mathrm{t}, 3 \mathrm{H}, \mathrm{OCH}_{2} \underline{\mathrm{CH}}_{3}\right) ;{ }^{13} \mathrm{C}-\mathrm{NMR} \delta 163(\mathrm{C}=\mathrm{O}), 152(\mathrm{HC}=)$, 
153, 147, 142, 127, 113, $111(\mathrm{Ph}), 116(\mathrm{CN}), 97(\mathrm{C}=), 61\left(\mathrm{OCH}_{2}\right)$, 56, 60, $61\left(\mathrm{PhOCH}_{3}\right)$, $14\left(\mathrm{OCH}_{2} \underline{\mathrm{CH}}_{3}\right) ; \mathrm{IR}\left(\mathrm{cm}^{-1}\right):$ 3022-2831 (m, C-H), $2224(\mathrm{~m}, \mathrm{CN}), 1726(\mathrm{~s}, \mathrm{C}=\mathrm{O}), 1586$ $(\mathrm{C}=\mathrm{C}), 1236\left(\mathrm{~s}, \mathrm{C}-\mathrm{O}-\mathrm{CH}_{3}\right), 841$ (s, C-H out of plane). Anal. Calcd. for $\mathrm{C}_{15} \mathrm{H}_{17} \mathrm{NO}_{5}$ : C,

61.85; H, 5.88; N, 4.81; Found: C, 61.84; H, 5.81; N, 4.76.

\subsection{Ethyl 2-cyano-3-(2,4,5-trimethoxylphenyl)-2-propenoate}

Yield 87\%; mp $163.1^{\circ} \mathrm{C},{ }^{1} \mathrm{H}-\mathrm{NMR} \delta 8.2$ (s, 1H, CH=), 7.1, 6.7 (m, 2H, Ph), 4.3 (q, 2H,

$\left.\mathrm{OCH}_{2}\right), 3.8\left(\mathrm{~s}, 9 \mathrm{H}, \mathrm{PhOCH}_{3}\right), 1.3\left(\mathrm{t}, 3 \mathrm{H}, \mathrm{OCH}_{2} \mathrm{CH}_{3}\right) ;{ }^{13} \mathrm{C}-\mathrm{NMR} \delta 163(\mathrm{C}=\mathrm{O}), 152(\mathrm{HC}=)$, 153, 146, 145, 114, 113, $97(\mathrm{Ph}), 116(\mathrm{CN}), 97(\mathrm{C}=), 61\left(\mathrm{OCH}_{2}\right), 56\left(\mathrm{PhOCH}_{3}\right), 14$

$\left(\mathrm{OCH}_{2} \underline{\mathrm{CH}}_{3}\right)$; IR $\left(\mathrm{cm}^{-1}\right): 3001-2848$ (m, C-H), 2223 (m, CN), 1716 (s, C=O), $1573(\mathrm{C}=\mathrm{C})$, 1239 (s, C-O-CH 3 ), 847 (s, C-H out of plane). Anal. Calcd. for $\mathrm{C}_{15} \mathrm{H}_{17} \mathrm{NO}_{5}: \mathrm{C}, 61.85 ; \mathrm{H}$, 5.88; N, 4.81; Found: C, 62.02; H, 5.70; N, 4.64.

\subsection{Ethyl 2-cyano-3-(2,4,6-trimethoxylphenyl)-2-propenoate}

Yield $87 \%$; mp $96.5^{\circ} \mathrm{C},{ }^{1} \mathrm{H}-\mathrm{NMR} \delta 8.1$ (s, $\left.1 \mathrm{H}, \mathrm{CH}=\right), 6.9$ (s, 2H, Ph), 4.3 (q, 2H, $\mathrm{OCH}_{2}$ ), $3.8\left(\mathrm{~s}, 9 \mathrm{H}, \mathrm{PhOCH}_{3}\right), 1.3\left(\mathrm{t}, 3 \mathrm{H}, \mathrm{OCH}_{2} \underline{\mathrm{H}}_{3}\right) ;{ }^{13} \mathrm{C}-\mathrm{NMR} \delta 163(\mathrm{C}=\mathrm{O}), 152(\mathrm{HC}=), 152$, 148, 144, 105, $93(\mathrm{Ph}), 116(\mathrm{CN}), 142(\mathrm{C}=), 61\left(\mathrm{OCH}_{2}\right), 56,55\left(\mathrm{PhOCH}_{3}\right), 14$ $\left(\mathrm{OCH}_{2} \underline{\mathrm{CH}}_{3}\right)$; IR $\left(\mathrm{cm}^{-1}\right):$ 3008-2839 (m, C-H), 2222 (m, CN), 1748 (s, C=O), 1589 (C=C), 1212 (s, C-O-CH3), 845 (s, C-H out of plane). Anal. Calcd. for $\mathrm{C}_{15} \mathrm{H}_{17} \mathrm{NO}_{5}: \mathrm{C}, 61.85 ; \mathrm{H}$, 5.88; N, 4.81; Found: C, 61.81; H, 5.83; N, 4.79.

\subsection{Ethyl 2-cyano-3-(3,4,5-trimethoxylphenyl)-2-propenoate}

Yield 77\%; mp $84.6^{\circ} \mathrm{C},{ }^{1} \mathrm{H}-\mathrm{NMR} \delta 8.2(\mathrm{~s}, 1 \mathrm{H}, \mathrm{CH}=), 7.2(\mathrm{~s}, 2 \mathrm{H}, \mathrm{Ph}), 4.3\left(\mathrm{q}, 2 \mathrm{H}, \mathrm{OCH}_{2}\right)$, $3.8\left(\mathrm{~s}, 9 \mathrm{H}, \mathrm{PhOCH}_{3}\right), 1.3\left(\mathrm{t}, 3 \mathrm{H}, \mathrm{OCH}_{2} \underline{\mathrm{H}}_{3}\right) ;{ }^{13} \mathrm{C}-\mathrm{NMR} \delta 163(\mathrm{C}=\mathrm{O}), 155(\mathrm{HC}=), 153$, 
147, 129, $109(\mathrm{Ph}), 116(\mathrm{CN}), 100(\mathrm{C}=), 61\left(\mathrm{OCH}_{2}\right), 61,56\left(\mathrm{PhOCH}_{3}\right), 14\left(\mathrm{OCH}_{2} \mathrm{CH}_{3}\right)$; IR ( $\left.\mathrm{cm}^{-1}\right): 3008-2839(\mathrm{~m}, \mathrm{C}-\mathrm{H}), 2228(\mathrm{~m}, \mathrm{CN}), 1734(\mathrm{~s}, \mathrm{C}=\mathrm{O}), 1545(\mathrm{C}=\mathrm{C}), 1239$ (s, CO-CH 3 ), 847 (s, C-H out of plane). Anal. Calcd. for $\mathrm{C}_{15} \mathrm{H}_{17} \mathrm{NO}_{5}$ : C, 61.85; H, 5.88; N, 4.81; Found: C, 62.01; H, 5.82; N, 4.70.

\section{Copolymerization}

Copolymerization (Sch. 1) of VAC and the ring-substituted EOCP resulted in formation of copolymers (Table 1) with weight-average molecular masses 1.1 to $6.8 \mathrm{kD}$.

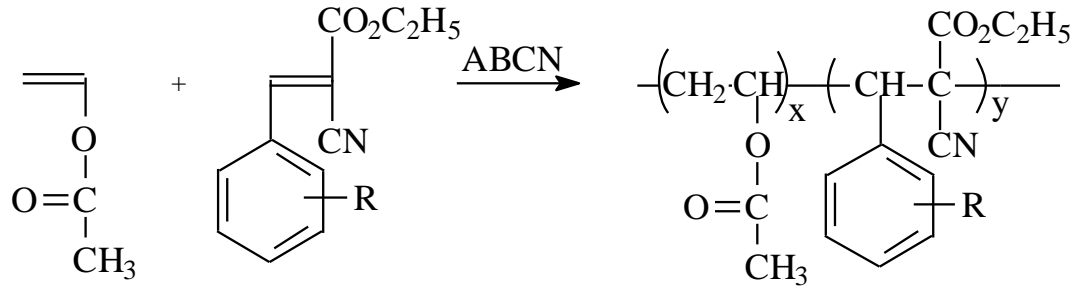

Scheme 1. Copolymerization of vinyl acetate and the ring-substituted ethyl 2-cyano-3phenyl-2-propenoates, $\mathrm{RPhCH}=\mathrm{C}(\mathrm{CN}) \mathrm{CO}_{2} \mathrm{C}_{2} \mathrm{H}_{5}$. $\mathrm{R}$ is 2-methoxy, 3-methoxy, 4-methoxy, 2-ethoxy, 4-ethoxy, 4-propoxy, 4-butoxy, 2,3-dimethoxy, 2,4-dimethoxy, 2,5-dimethoxy, 3,4-dimethoxy, 2,3,4-trimethoxy, 2,4,5-trimethoxy, 2,4,6-trimethoxy, 3,4,5-trimethoxy. According to the nitrogen elemental analysis, between 42.2 and 49.9 mol\% of TSE monomer is present in the copolymers prepared at $\mathrm{VAC} / \mathrm{EOCP}=3(\mathrm{~mol})$, which is indicative of relatively high reactivity of the monomers towards VAC. 
Table 1. Copolymerization of vinyl acetate and ring-substituted ethyl 2-cyano-3-phenyl2-propenoates, $\mathrm{RPhCH}=\mathrm{C}(\mathrm{CN}) \mathrm{CO}_{2} \mathrm{C}_{2} \mathrm{H}_{5}$.

\begin{tabular}{|c|c|c|c|c|c|c|c|c|c|}
\hline & & & & & & \multicolumn{4}{|c|}{$T G A$} \\
\hline $\mathrm{R}$ & $\begin{array}{l}\text { Yield }^{\mathrm{a}} \\
\mathrm{wt} \%\end{array}$ & $\begin{array}{c}\mathrm{N} \\
\mathrm{wt} \%\end{array}$ & $\begin{array}{l}\text { EOCP } \\
\text { in } \\
\text { pol., } \\
\text { mol\% }\end{array}$ & $\begin{array}{l}\mathrm{Mw}_{\mathrm{W}} \\
\mathrm{kD}\end{array}$ & $\begin{array}{l}\mathrm{T}_{\mathrm{g}} \\
{ }^{\circ} \mathrm{C}\end{array}$ & $\begin{array}{c}\text { Onset of } \\
\text { decomp. } \\
{ }^{\circ} \mathrm{C}\end{array}$ & $\begin{array}{c}10 \% \\
\mathrm{wt} \\
\text { loss, } \\
{ }^{\circ} \mathrm{C} \\
\end{array}$ & $\begin{array}{l}50 \% \\
\mathrm{wt} \\
\text { loss, } \\
{ }^{\circ} \mathrm{C} \\
\end{array}$ & $\begin{array}{c}\text { Residue } \\
\text { at } \\
500^{\circ} \mathrm{C}, \\
\mathrm{wt}^{\circ} \%\end{array}$ \\
\hline 2-Methoxy & 52.3 & 4.35 & 48.6 & 5.6 & 88 & 196 & 262 & 341 & 15.7 \\
\hline 3-Methoxy & 38.9 & 4.28 & 47.2 & 6.8 & 86 & 166 & 225 & 334 & 12.6 \\
\hline 4-Methoxy & 58.2 & 4.29 & 47.4 & 6.2 & 88 & 179 & 269 & 340 & 15.7 \\
\hline 2-Ethoxy & 56.2 & 4.21 & 49.6 & 6.4 & 91 & 163 & 257 & 338 & 14.2 \\
\hline 4-Ethoxy & 39.3 & 4.19 & 49.1 & 4.1 & 86 & 149 & 209 & 332 & 15.7 \\
\hline 4-Propoxy & 69.2 & 3.96 & 47.6 & 4.8 & 88 & 206 & 296 & 343 & 15.4 \\
\hline 4-Butoxy & 70.2 & 3.62 & 42.2 & 4.5 & 78 & 189 & 256 & 329 & 14.8 \\
\hline 2,3-Dimethoxy & 54.3 & 3.92 & 47.2 & 1.1 & 111 & 149 & 209 & 333 & 25.9 \\
\hline 2,4-Dimethoxy & 78.4 & 3.91 & 46.9 & 1.4 & 112 & 187 & 241 & 286 & 4.7 \\
\hline 2,5-Dimethoxy & 34.2 & 3.97 & 48.4 & 1.7 & 141 & 179 & 224 & 337 & 25.3 \\
\hline 3,4-Dimethoxy & 47.2 & 3.99 & 48.9 & 2.2 & 117 & 173 & 223 & 267 & 4.1 \\
\hline $\begin{array}{l}\text { 2,3,4- } \\
\text { Trimethoxy }\end{array}$ & 56.3 & 3.65 & 48.2 & 1.5 & 154 & 187 & 236 & 316 & 8.1 \\
\hline $\begin{array}{l}2,4,5- \\
\text { Trimethoxy }\end{array}$ & 67.3 & 3.62 & 47.3 & 1.2 & 104 & 179 & 227 & 306 & 7.3 \\
\hline $\begin{array}{l}2,4,6- \\
\text { Trimethoxy }\end{array}$ & 65.3 & 3.63 & 47.6 & 2.7 & 98 & 181 & 251 & 292 & 2.2 \\
\hline $\begin{array}{l}3,4,5- \\
\text { Trimethoxy }\end{array}$ & 48.3 & 3.65 & 48.2 & 3.6 & 110 & 172 & 231 & 302 & 5.8 \\
\hline
\end{tabular}




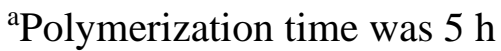

According to the nitrogen elemental analysis, between 42.2 and $49.6 \mathrm{~mol} \%$ of TSE monomer is present in the copolymers prepared at VAC/EOCP $=3(\mathrm{~mol})$, which is indicative of relatively high reactivity of the monomers towards VAC.

\section{Structure and Thermal Properties}

The structure of VAC-EOCP copolymers was characterized by IR and NMR spectroscopy. A comparison of the spectra of the monomers, copolymers, and polyvinyl acetate with the spectra of ring-unsubstituted ethyl 2-cyano-3-phenyl-2-propenoate - VAC [28] shows, that the reaction between the EOCP monomers and VAC is a copolymerization.

The copolymers prepared in the present work are all soluble in ethyl acetate, THF, DMF and $\mathrm{CHCl}_{3}$ and insoluble in methanol, ethyl ether, and petroleum ether. They are amorphous and show no crystalline DSC endotherm. Results of thermal analysis of VACEOCP copolymers are presented in Table 1 . Relatively high $T_{g}$ of the copolymers (78-154 ${ }^{\circ} \mathrm{C}$ ) in comparison with that of polyvinyl acetate, $T_{g}=28-31^{\circ} \mathrm{C}$ [29] indicates decrease of chain mobility of the copolymer due to the high dipolar character of the EOCP structural units.

Information on the degradation of the copolymers was obtained from thermogravimetric analysis. Decomposition of the copolymers in nitrogen occurred in two steps, apparently acetic acid elimination [30] in $160-350^{\circ} \mathrm{C}$ range followed by more slow decomposition of formed residue (25.9-2.2 wt\%), which then decomposed in the 500-650 ${ }^{\circ} \mathrm{C}$ range. 


\section{Conclusions}

Oxy ring-substituted ethyl 2-cyano-3-phenyl-2-propenoates were prepared and copolymerized with vinyl acetate. The compositions of the copolymers were calculated from nitrogen analysis and the structures were analyzed by IR, $\mathrm{H}^{1}$ and ${ }^{13} \mathrm{C}-\mathrm{NMR}$. Thermal gravimetric analysis indicated that the copolymers decompose in in two steps, first in the $200-500^{\circ} \mathrm{C}$ range with residue (13-16 wt $\%$ ), which then decomposed in the $500-650^{\circ} \mathrm{C}$ range.

\section{Acknowledgments}

The authors are grateful to acknowledge that the project was partly supported by the Coatings Industry Education Fund and Chicago Society of Coating Technology.

\section{References}

[1] Nucleophilic attacks on carbon-carbon double bonds. IV. Cis-trans isomerization of ethyl $\alpha$-cyano- $\beta$-(o-methoxyphenyl)acrylate by various nucleophiles. Patai, Saul; Rappoport, Zvi. Journal of the Chemical Society (1962), 396-403.

[2] Glutathione-S-transferase activity in Setaria cervi females and effect of new antifilarial compounds. Srivastava, Arvind K.; Tripathi, R. P.; Khan, A. R.; Bhaduri, A. P.; Singh, S. N.; Chatterjee, R. K. Indian Journal of Parasitology (1994), 18(2), 127-133.

[3] Nitriles in heterocyclic synthesis: novel synthesis of benzo[c]coumarin and of benzo[c]pyrano[3,2-c]quinoline derivatives. Hafez, Ebtisam Abdel Aziz; Elnagdi, Mohamed 
Hilmy; Elagamey, Abdel Ghani Ali; El-Taweel, Fathy Mohamed Abdel Aziz. Heterocycles (1987), 26(4), 903-7.

[4] Fabrication of electroluminescent device using organic crystals. Hatasawa, Takenobu; Hidaka, Takahiro. Japan, JP05182763 A 1993-07-23.

[5] Stereochemistry of interaction of pyridinium ylides with $\alpha, \beta$-unsaturated nitriles.

Shestopalov, A. M.; Sharanin, Yu. A.; Litvinov, V. P. Khimiya Geterotsiklicheskikh Soedinenii (1990), (3), 363-9.

[6] Sustainable C-C bond formation through Knoevenagel reaction catalyzed by MgO-based catalysts. Alvarez, Lucrecia; Hidalgo-Carrillo, Jesus; Marinas, Alberto; Marinas, Jose Maria; Urbano, Francisco J. Reaction Kinetics, Mechanisms and Catalysis (2016), 118(1), 247-265.

[7] Application of ethyl p-methoxycinnamate and derivatives in maintaining self-renewal and pluripotency of stem cells. Bao, Yongli; Li, Yuxin; Qin, Hongshuang World Intellectual Property Organization, WO2016119085 A1 2016-08-04.

[8] DBU-mediated [4 + 2] annulations of donor-acceptor cyclopropanes with 3-aryl-2cyanoacrylates for the synthesis of fully substituted anilines. Liu, Jiaming; Qian, Siran; Su, Zhenjie; Wang, Cunde. RSC Advances (2017), 7(61), 38342-38349.

[9] Azaindazoles as Btk kinase modulators and their preparation. Duan, Jingwu; Jiang, Bin; Lu, Zhonghui. World Intellectual Property Organization, WO2011019780 A1 2011-02-17. Database: CAplus.

[10] Novel Copolymers of Alkyl and Alkoxy Ring-Substituted Ethyl 2-Cyano-3-phenyl-2propenoates and Styrene. Kharas, Gregory B.; Diener, Celeste A.; Barbarawi, Hadeal A.; 
Beavers, Nicole D.; Borovilos, Maria; Carney, Jason; Fox, Ashley A.; McClelland, Kristen M.; Gehle, Jessica L.; Yedlinski, Jason; et al. Journal of Macromolecular Science, Pure and Applied Chemistry (2004), A41(8), 889-896.

[11] Novel Copolymers of Styrene. 5. Oxy Ring-Substituted Ethyl 2-Cyano-3-Phenyl-2Propenoates. Kharas, Gregory B.; Delgado, Alexander A.; Gange, Nicole; Hartzell, Matthew C.; Hawley, Nicholas W.; Kupczyk, Kathryn A.; Lam, Emily K.; Lyngaas, Stephanie S.; Mohammad, Fahad B.; Montgomery, Mary E.; et al. J. Macromol. Sci., Part A: Pure and Applied Chemistry (2013), 50(3), 271-275.9.

[12] Highly efficient synthesis of pyranoquinoline derivatives catalyzed by piperidine.

Kaur, Jasreen; Bariwal, Jatinder; Bedi, Preet Mohinder Singh; Kaur, Maninderjit;

Kapoor, Amit; Kaur, Mandeep; Verma, Poonam. World Journal of Pharmacy and Pharmaceutical Sciences (2017), 6(12), 850-870.

[13] Design, Synthesis and Anticancer Activity of Novel Benzothiazole Analogues. Hassan, Aisha Y.; Sarg, Marwa T.; Hussein, Ebtehal M. Journal of Heterocyclic Chemistry (2019), 56(4), 1437-1457.

[14] Synthesis and antimicrobial activity of some novel hydrazide, benzochromenone, dihydropyridine, pyrrole, thiazole and thiophene derivatives. Refat, Hala M.; Fadda, A.

A. European Journal of Medicinal Chemistry (2013), 70, 419-426.

[15] Novel Polyfunctional Pyridines as Anticancer and Antioxidant Agents. Synthesis, Biological Evaluation and in Silico ADME-T Study. Badr, Mona Hany; Rostom, Sherif Ahmed Fawzi; Radwan, Mohammed Fouad. Chemical \& Pharmaceutical Bulletin (2017), $65(5), 442-454$. 
[16] Synthesis and biological screening of some novel 3-amino-4-arylidene-5pyrazolones and thiazolo[3,2-a]pyrimidines. Oza, Tanvi D.; Parikh, Arun; Gudaparthi, Vijayalakshmi. Pharma Science Monitor (2012), 3(4 (Suppl. 2)), 2775-2785.

[17] Antitumor activity of novel pyridine, thiophene and thiazole derivatives. Ghorab, Mostafa M.; Al-Said, Mansour S. Archives of Pharmacal Research (2012), 35(6), 965973.

[18] Synthesis, characterization and heterogeneous base catalysis of amino functionalized lanthanide metal-organic frameworks. Huang, Jinping; Li, Chunmei; Tao, Lingling; Zhu, Huilin; Hu, Gang. Journal of Molecular Structure (2017), 1146, 853-860.

[19] Synthesis, antimicrobial and antitumor study of new pyrido[2,1-a]isoquinolines via isoquinoline-1-acetonitrile. Teleb, Mohamed A. M.; Hassaneen, Hamdi M.; Abdelhadi, Hyam A.; Laboud, Yara N.; Saleh, Fatma M. Heterocycles (2021), 102(9), 1729-1742. [20] Synthesis and Copolymerization of Ring-substituted Ethyl 2-cyano-3-phenyl-2propenoates with Styrene. G.B. Kharas, H.A. Barbarawi, N.D. Beavers, M.Borovilos, J. Carney, E. Cygan, C.A. Diener, A.A. Fox, J. Gibson, K.M. McClelland, S. Mills, N. Tuzik, J. Yedlinski, and K. Watson, Division of Polymer Chemistry, 224th ACS National Meeting, Boston, MA, August 18-22, 2002. Polymer Preprints 2002, 43(2), 1120.

[21] Novel copolymers of Alkyl and Alkoxy Ring-substituted 2-Cyano-3-phenyl-2propenoates and Styrene. G.B. Kharas, C.A. Diener, H.A. Barbarawi, N.D. Beavers, M. Borovilos, J. Carney, A.A. Fox, K.M. McClelland, J. Yedlinski, and K. Watson. J. Macromol. Sci., A41 (8), 889-896 (2004) 
[22] Novel styrene copolymers of some ethyl phenylcyanoacrylates. Raven-Kiera M.

Cupid, Reese C. Leitao, Josiah D. Stewart, Michael J. Turski, Sara M. Rocus, William S.

Schjerven, and Gregory B. Kharas. ChemRxiv. Cambridge Open Engage Version 1 Aug

26, 2021. https://doi.org/10.33774/chemrxiv-2021-kkn25

[23] Novel Copolymers of Styrene. 5. Oxy Ring-substituted Ethyl 2-Cyano-3-Phenyl-2-

propenoates. G.B. Kharas, A.A. Delgado, N. Gange, M.C. Hattzell, N.W. Hawley, K.A.

Kupczyk, E.K. Lam, S.S. Lyngaas, F.B. Mohammad, M.E. Montgomery, A.J. Ryan, and

V.M. Wright. J. Macromol. Sci. A50 (3) 271-275 (2013).

[24] Novel Copolymers of Styrene. 6. Alkoxy Ring-Substituted Ethyl 2-Cyano-3-Phenyl-2

Propenoates. Kharas, Gregory B.; Delgado, Alexander A.; Anderson, Nathan E.;

Bajor, Agnieszka; Colbert, Acheera C.; Coleman, Adrienne; Gregory, Carine E.;

Hayes, John; Lantin, Jason; Malecki, Joseph M.; et al. Journal of Macromolecular

Science, Part A: Pure and Applied Chemistry (2013), 50(3), 276-280.

[25] SciFinder structure search accessed Dec 16, 2021.

[26] Novel functional copolymers of vinyl acetate: 1. Alkyl ring-substituted ethyl 2cyano-3-phenyl-2-propenoates. Benjamin Y. Killam, Rima T. Barkauskas, Daniel P. Dembiec, Rebecca S. Farrell, Maritza A. Gallego, Kelly A. Kaiser, Michelle L. Keeling, Kari Y. Kang, Gretchen R. Verdoorn, and Gregory B. Kharas. ChemRxiv. Cambridge Open Engage Version 1 Sep 06, 2021. https://doi.org/10.33774/chemrxiv-2021-ksld6

[27] Smith, M. B., March, J. (2001) Addition to Carbon-Hetero Multiple Bonds, In March's Advanced Organic Chemistry; J. Wiley \& Sons: New York, Ch.16, 1225. 
[28] Odian, G. Principles of Polymerization, 4th Ed., Wiley-Interscience: New York, 2004.

[29] Characterization of copolymers of vinyl acetate with ethyl $\alpha$-cyanocinnamate. G.B.

Kharas and D.H. Kohn. J. Polym. Sci. Polym. Chem. Ed., 23, 577-582 (1984).

[30] Characterization and physical properties of low molecular weight poly(vinyl acetate) and poly(vinyl alcohol). Toshiaki Sato and Takuji Okaya. Polymer Journal, Vol. 24, No.9, pp 849-856 (1992).

[31] The thermal degradation of poly(vinyl acetate) and poly(ethylene-co-vinyl acetate), Part I: Experimental study of the degradation mechanism. B. Rimez, H. Rahier, G. Van Assche, T. Artoos, M. Biesemans, B. Van Mele. Polymer Degradation and Stability, 93 (2008) 800-810. 